\title{
Genome-wide identification, classification, and analysis of two-component signal system genes in maize
}

\author{
Z.X. Chu*, Q. Ma*, Y.X. Lin, X.L. Tang, Y.Q. Zhou, S.W. Zhu, J. Fan and \\ B.J. Cheng
}

Anhui Province Key Laboratory of Crop Biology, School of Life Science, Anhui Agricultural University, Hefei, China

*These authors contributed equally to this study.

Corresponding authors: B.J. Cheng / J. Fan

E-mail: beijiucheng@ahau.edu.cn / fanjun@ahau.edu.cn

Genet. Mol. Res. 10 (4): 3316-3330 (2011)

Received March 23, 2011

Accepted August 31, 2011

Published December 8, 2011

DOI http://dx.doi.org/10.4238/2011.December.8.3

\begin{abstract}
Cytokinins play many vital roles in plant development and physiology. In plants, cytokinin signals are sensed and transduced by the two-component signal system. This signaling cascade is typically composed of three proteins: a sensory histidine kinase, a histidine phosphotransfer protein, and a response regulator. Through a comprehensive genomewide analysis of the maize (Zea mays) genome, 48 genes were identified, including $11 \mathrm{ZmHKs}, 9 \mathrm{ZmHPs}$, and $28 \mathrm{ZmRRs}$ (21 A-type ZmRRs and 7 B-type $Z m R R$ s). Using maize genome sequence databases, we analyzed conserved protein motifs and established phylogenetic relationships based on gene structure, homology, and chromosomal location. The duplication of these two-component system genes in the maize genome corresponded to the clusters of these genes in the phylogenetic trees. Sequence analysis of the duplicate genes demonstrated that one gene may be in gene duplication, and that there was significant variation in the evolutionary history of the different gene families. We assessed the expression levels of all $Z m R R \mathrm{~s}$ in the leaves and roots by reverse transcription PCR; they were all found to be active. Our results provide a foundation for functional and evolutionary
\end{abstract}


studies on maize two-component signal system proteins.

Key words: Maize (Zea mays); Two-component signal system; Phylogenetic analysis; Duplication; Cytokinin; RT-PCR

\section{INTRODUCTION}

Plant cytokinins play important roles in many different developmental and physiological processes (Martín et al., 2000; Mok and Mok, 2001; Forde, 2002). The two-component system (TCS) mediates cytokinin signal transduction in bacterium, fungi, and plants, and it is vital for organizing the response to changes in environmental conditions, nutrients, oxygen, light, and osmotic pressure (Yamada and Shiro, 2008).

The TCSs have been studied most extensively in plants and bacterium (Hutchison and Kieber, 2002; Asakura et al., 2003; Yonekura-Sakakibara et al., 2004). Originally, the His-Asp phosphorelay system was referred to as a two-component regulatory system in bacterium, and typically is comprised of two proteins: a sensor and a response regulator (Stock et al., 2000; West and Stock, 2001). In this signal transduction pathway, the membrane receptor senses the signals (cytokinins) by phosphorylating its own conserved histidine acid residues (His). Then, the phosphoryl group is transmitted to a conserved Aspartic acid residue (Asp) in the receiver domain of a cognate response regulator to influence gene transcription (Stock et al., 2000; Thomason and Kay, 2000).

Eukaryotes like yeast and plants have evolved a more complex multi-step TCS system with additional phosphorylation steps (Grefen and Harter, 2004) and histidine phosphotransfer proteins. In the multi-step two-component system, the phosphoryl group is transferred from His to an Asp residue in the C-terminal receiver domain of the hybrid receptor-histidine kinase. Histidine phosphotransfer proteins transfer the phosphoryl group from the receiver domain of the receptor-histidine kinase to the receiver domain within the response regulator.

The TCS has been studied extensively in Arabidopsis. In this model plant, there are $3 H K$ genes (AHK2, AHK3 and AHK4/CRE1/WOL), 6 HP genes (AHP1-6) and $24 R R$ genes (ARR1-24) (Mahonen AP et al., 2000; Inoue et al., 2001; Suzuki et al., 2001). Histidine kinases play an important role in the developmental processes of shoot growth, leaf senescence, seed size, germination, root development, and cytokinin metabolism (Riefler et al., 2006). A hybrid histidine kinase, CKI1, was first isolated in the absence of exogenous cytokinin in Arabidopsis (Kakimoto, 1996). A loss of the function allele of CKII confers a loss of function that is transmitted through the female gamete (Pischke et al., 2002). However, the CKI1 histidine kinase is not considered to be a cytokinin receptor because it does not require cytokinins for its activity. By using heterologous yeast and $E$. coli systems, $A H K 4 / C R E 1 / W O L$ was isolated; it was proposed to be a cytokinin receptor that exhibited reduced response to cytokinins in the mutant (Inoue et al., 2001; Suzuki et al., 2001; Ueguchi et al., 2001). All AHKs share a CHASE domain (cyclase/histidine kinase-associated sensory extracellular), which is the putative recognition site for cytokinins (Ueguchi et al., 2001).

The AHPS code for $\sim 12 \mathrm{kDa}$ proteins. They contain the highly conserved XHQXKGSSXS motif that mediates the transfer of a phosphate group from the receiver domain of an AHK to the receiver domain of an ARR in a multi-step phosphorelay signal transduction pathway (Hwang et al., 2002).

The ARRs have been found characterized by the DDK domain that accepts the phosphoryl group (Schaller et al., 2002). Most of response regulators are transcription factors with a receiver domain in the N-terminal (Aoyama and Oka, 2003). Phosphorylation of response regulators in- 
duces a change in the output domain to activate or deactivate transcription and other biological processes (Stock et al., 2000; West and Stock, 2001; Hass et al., 2004). ARRs can be divided into two major classes, type A and type B, according to the amino-acid sequence and conserved domains. Type-A ARRs are primary cytokinin response genes. They are relatively small and contain a receiver domain along with short C-terminal extensions. The structure of the type-AARRs is similar to CheY (D'Agostino and Kieber, 1999). Type-A ARRs show considerable heterogeneity among isoforms; 60 to $93 \%$ of the amino acids differ, with much greater regional heterogeneity within the C-terminal extensions than within the receiver domain (where there is $70 \%$ sequence homology). In general, type-B ARRs display 70\% amino acid sequence homology (D'Agostino et al., 2000).

Type-B ARRs contain a receiver domain and a large C-terminal output domain containing a GARP motif (Lohrmann et al., 1999) which is related to the Myb repeat of transcription factors (Riechmann et al., 2000; Sakai et al., 2000). The C-terminal region of type-B ARRs contains nuclear localization sequences and P/Q-rich regions similar to other transcription factors (Lohrmann et al., 1999). Indeed, type-B ARRs have bee found in the nucleus (Sakai et al., 2000; Hwang and Sheen, 2001; Lohrmann et al., 2001; Hwang et al., 2002). Type-B ARR mutants showed decreased sensitivity to cytokinin, resulting in defects in root elongation, lateral root formation, callus induction, and greening. Type-B ARRs cause induction of cytokinin primary response genes (Mason et al., 2005).

Five AHKs, 6 AHPs, and 24 ARRs have been identified in Arabidopsis while 5 OsHKs, $5 O s H P s$, and 9 OsRRs have been identified in rice. There has been relatively little research on the cytokinin two-component system of maize. The genome sequence of Zea mays has been completed, allowing for a more detailed genomic and proteomic analysis. Studying this signaling system is significant in physiological of the development of plant and lays a foundation for the study of signal transduction mechanisms.

\section{MATERIAL AND METHODS}

\section{Zea mays genome database}

The completed genome sequence of Zea mays was downloaded from the maize sequence database (http://www.maizesequence.org/index.html).

\section{Bioinformatics methods}

\section{Sequence retrieval}

Sequences of 3 AHKs, 6 AHPs, and 24 ARRs from Arabidopsis and 5 OsHKs, 2 OsHPs and 9 OsRRs from rice were downloaded from the NCBI databases. Using these sequences as queries in BLASTN, we searched for putative genes encoding TCS genes in the Zea mays genomes with the DNATOOLS software (version 6.0, http://www.dnatools.com/). The threshold expectation value was set to $10^{-3}$, which was crucial to find the maximum number of putative genes. By using the Pfam database (http://pfam.janelia.org/), all of the sequences which meet the requirements were analyzed to eliminate genes that did not contain the known conserved domains and motifs, including the CHASE domain, HisKA (phosphoacceptor) domain, HATPase (histidine kinase-like ATPase domain) domain, Hpt (histidine-containing phosphotransfer) domain, and RR (response regulate) domain. Then the utility ClustalX (Thompson et al., 1994) of MEGA software (version 4.0; http://www.megasoftware. net/) was used to eliminate identical sequences which located within longer sequences or genes. 


\section{Sequence alignment}

The online MEME (Multiple EM for Motif Elicitation) utility was used to identify motifs of ZmHKs, ZmHPs, and ZmRRs with an expected value lower than $2 \times 10^{-30}$ (http:// meme.sdsc.edu; Bailey et al., 2006). The CBS database (http://www.cbs.dtu.dk/services/ TMHMM/) was used to uncover the transmembrane domains of ZmHKs. Subsequently, the Pfam database was used to search the amino acid sequences of conserved domains. Then the amino acid sequences were analyzed by ClustalX (version 1.83) to determine homology.

\section{Phylogenetic analysis and chromosomal locations of TCS genes}

Phylogenetic analysis of the sequences was conducted using MEGA (version 4.0) and with the Bootstrap neighbor-joining method. Confidence limits of each branch in the phylogeny tree were assessed by 1000 bootstrap replications and expressed as percentage values. The starting position of all TCS genes was confirmed by tBLASTn search $(\mathrm{P}=0.001)$ using a local database of complete maize genome sequences for each chromosome. MapInspect software (http://www.plantbreeding.wur.nl/uk/software_mapinspect.html) was subsequently used for a graphic portrayal of Zea mays two-component genes. Gene duplication events were also investigated by evolutipon distance calculation. ClustalX (version 1.83) was used again to align amino acid sequences of two-component proteins and calculate their evolutionary distance.

\section{RT-PCR analysis}

Seeds of $\mathrm{B} 73$ were germinated on plates at $37^{\circ} \mathrm{C}$ for two days. Seedlings were then transferred to a nutrient solution at $28^{\circ} \mathrm{C}$ in a culture room under a 16:8-h light-dark photoperiod for an additional 15 days. Total RNA was extracted from the roots and leaves of seedlings using an RNAiso reagent (TaKaRa Biotechnology, Otsu, Japan) according to manufacturer instructions. After RNasefree DNase treatment (TaKaRa Biotechnology, Japan), purified RNA was reverse transcribed using the Access RT-PCR reverse transcription-polymerase chain reaction system (Promega, USA) to obtain first-strand cDNA. Twenty-eight genes were detected for analyzing their expression in leaves and roots. The gene-specific primers of these 28 genes were designed based on the sequences of the receiver domain. Reactions were performed with Taq Polymerase (Takara Biotechnology, Japan) on a thermal cycler (Supplemetary Table 1) (Tpersonal 48; Biometra, Göttingen, Germany), with the following profile: initial denaturation at $94^{\circ} \mathrm{C}$ for $5 \mathrm{~min}, 30$ cycles of denaturation at $94^{\circ} \mathrm{C}$ for $30 \mathrm{~s}$, annealing at $50^{\circ} \mathrm{C}$ (changed by $-1{ }^{\circ} \mathrm{C}$ per cycle) for $30 \mathrm{~s}$, polymerization at $72^{\circ} \mathrm{C}$ for $45 \mathrm{~s}$, and final elongation at $72^{\circ} \mathrm{C}$ for $10 \mathrm{~min}$.

\section{RESULTS}

\section{Identification and classification of TCS genes in Zea mays}

Seventy-two putative genes of the maize cytokinin two-component signaling system were initially identified. Twenty-four genes were excluded by searching the Pfam database. Eleven ZmHKs with highly conserved CHASE domain were identified that could be divided into two groups, $\mathrm{ZmHK}$ and ZmHKL (ZmHK-like), according to the presence or absence of the HisKA, HATPase, or RD domains. 
Nine ZmHPs with highly conserved HPt domains and 28 ZmRRs with highly conserved receiver domains were identified. The $28 \mathrm{ZmRRs}$ could be divided into two types, A-type ZmRR and B-type ZmRR, according to whether they contained the Myb DNA-binding domain. In Table 1, we present a summary that includes gene name, chromosome location, protein length, and type for each predicted cytokinin two-component signaling gene in maize. The average amino acid length of ZmHKs, ZmHPs, A-type ZmRRs, and B-type ZmRRs were 860 aa, 170 aa, 259 aa, and 577 aa, respectively.

Table 1. Features of the cytokinin two-component signaling system genes in maize. The accession numbers of the full-length cDNA sequence are available at NCBI (NF, not found).

\begin{tabular}{|c|c|c|c|c|c|}
\hline Gene & Gene name & Chromosome & cM position & Length (aa) & Type \\
\hline \multicolumn{6}{|c|}{ Maize cytokinin receptors } \\
\hline $\mathrm{ZmHK} 1$ & GRMZM2G158252_T01 & 5 & 38 & 940 & \\
\hline $\mathrm{ZmHK} 2$ & GRMZM2G423456_T02 & 8 & 160 & 1007 & \\
\hline $\mathrm{ZmHK} 3$ & GRMZM2G155767-T01 & 1 & 268 & 997 & \\
\hline $\mathrm{ZmHK} 4$ & GRMZM2G039696_T02 & 5 & 10.2 & 996 & \\
\hline $\mathrm{ZmHK} 5$ & GRMZM2G471529_T02 & 3 & 157.6 & 1007 & \\
\hline ZmHK6 & GRMZM2G125943 T01 & 4 & 166.4 & 975 & \\
\hline $\mathrm{ZmHK} 7$ & GRMZM2G151223 T02 & 5 & 204.5 & 974 & \\
\hline $\mathrm{ZmHK} 8$ & GRMZM2G039696 T01 & 5 & 10.2 & 966 & \\
\hline ZmHKL1 & GRMZM2G158252 T03 & 5 & 38 & 703 & \\
\hline $\mathrm{ZmHKL} 2$ & GRMZM2G151223 T01 & 5 & 204.5 & 505 & \\
\hline ZmHKL3 & GRMZM2G039696 T03 & 5 & 10.2 & 387 & \\
\hline \multicolumn{6}{|c|}{ Maize histidine phosphotransfer protein gene family } \\
\hline $\mathrm{ZmHP} 1$ & GRMZM2G451604_T01 & 4 & 60.2 & 200 & \\
\hline $\mathrm{ZmHP} 2$ & GRMZM2G014154_T03 & 2 & 162.5 & 304 & \\
\hline $\mathrm{ZmHP} 3$ & GRMZM2G016439-T01 & 1 & 204.6 & 144 & \\
\hline $\mathrm{ZmHP} 4$ & GRMZM2G451604_T03 & 4 & 60.2 & 144 & \\
\hline ZmHP5 & GRMZM2G124890_T01 & 6 & 130.4 & 148 & \\
\hline $\mathrm{ZmHP6}$ & GRMZM2G039246_T01 & 3 & 197.2 & 153 & \\
\hline $\mathrm{ZmHP7}$ & GRMZM2G016439 T02 & 1 & 204.6 & 133 & \\
\hline $\mathrm{ZmHP8}$ & GRMZM2G173710_T02 & 8 & 123.1 & 152 & \\
\hline $\mathrm{ZmHP9}$ & GRMZM2G451604 T02 & 4 & 60.2 & 154 & \\
\hline \multicolumn{6}{|c|}{ Maize response regulator genes } \\
\hline ZmRR1 & GRMZM2G129954_T01 & 10 & 1.2 & 262 & A \\
\hline $\mathrm{ZmRR} 2$ & GRMZM2G319187 T01 & 8 & 156.6 & 279 & A \\
\hline ZmRR3 & GRMZM2G392101_T01 & 10 & 147.3 & 159 & A \\
\hline $\mathrm{ZmRR} 4$ & GRMZM2G040736_T01 & 2 & 2 & 156 & A \\
\hline ZmRR5 & GRMZM2G148056_T01 & 7 & 42.8 & 148 & A \\
\hline ZmRR6 & GRMZM2G096171 T01 & 4 & 116.8 & 242 & $\mathrm{~A}$ \\
\hline ZmRR7 & GRMZM2G156019_T01 & 3 & 144.4 & 123 & $\mathrm{~A}$ \\
\hline ZmRR8 & GRMZM2G179827_T01 & 2 & 47.7 & 244 & $\mathrm{~A}$ \\
\hline ZmRR9 & GRMZM2G319187_T02 & 8 & 156.6 & 173 & $\mathrm{~A}$ \\
\hline ZmRR10 & GRMZM2G016145_T01 & 4 & 77.8 & 124 & $\mathrm{~A}$ \\
\hline ZmRR11 & GRMZM2G319187_T03 & 8 & 156.6 & 144 & $\mathrm{~A}$ \\
\hline ZmRR12 & GRMZM2G005732_T02 & 7 & 169.1 & 177 & $\mathrm{~A}$ \\
\hline ZmRR13 & GRMZM2G033962 T01 & 2 & 215.7 & 657 & $\mathrm{~A}$ \\
\hline ZmRR14 & GRMZM2G095727_T05 & 9 & 134.2 & 766 & A \\
\hline ZmRR15 & GRMZM2G179024 T01 & 7 & 137.5 & 629 & A \\
\hline ZmRR16 & GRMZM2G020081_T01 & 4 & 139.8 & 515 & $\mathrm{~A}$ \\
\hline ZmRR17 & GRMZM2G308046_T01 & 2 & 101.7 & 187 & $\mathrm{~A}$ \\
\hline ZmRR18 & GRMZM2G099797_T04 & 1 & 28.5 & 114 & $\mathrm{~A}$ \\
\hline ZmRR19 & GRMZM2G401821_T01 & 6 & 98.6 & 106 & $\mathrm{~A}$ \\
\hline ZmRR20 & GRMZM2G013612-T01 & 2 & 80.6 & 225 & $\mathrm{~A}$ \\
\hline ZmRR21 & GRMZM2G460594_T01 & 1 & 273.9 & 88 & $\mathrm{~A}$ \\
\hline $\mathrm{ZmRR} 22$ & GRMZM2G177220_T01 & 3 & 164.3 & 584 & B \\
\hline ZmRR23 & GRMZM2G100318_T01 & 8 & 162.2 & 585 & B \\
\hline ZmRR24 & GRMZM2G360523 T01 & 9 & 98 & 671 & B \\
\hline ZmRR25 & GRMZM2G479110_T01 & 5 & 212.9 & 676 & $\mathrm{~B}$ \\
\hline ZmRR26 & GRMZM2G099797 T01 & 1 & 28.5 & 684 & B \\
\hline $\mathrm{ZmRR} 27$ & GRMZM2G409974 T01 & 9 & 13.3 & 669 & B \\
\hline ZmRR28 & GRMZM2G126834_T02 & 9 & 141.4 & 656 & $\mathrm{~B}$ \\
\hline
\end{tabular}




\section{Sequence alignments and analysis}

The $8 \mathrm{ZmHK}$ proteins all possessed three conserved domains (CHASE domain, transmitter domain, and receiver domain) as determined by the Pfam database. Similarly, each of the $3 \mathrm{ZmHKL}$ proteins contained a CHASE domain but no integral transmitter domain or receiver domain. According to the CBS database, the ZmHK8 protein did not a contain transmembrane domain, while ZmHK1, ZmHK6, ZmHK7, ZmHKL1, and ZmHKL2 all contained one transmembrane domain, and ZmHK2-5 and ZmHKL3 contained two transmembrane domains. These results illustrate that the CHASE domain is highly conserved in the evolution of ZmHKs, while the HisKA domain and HATPase domain are present in almost all ZmHK receiver domains. In contrast, transmembrane domains are not well conserved. These outcomes also predict that the CHASE domain is necessary for recognizing specific cytokinins. All $9 \mathrm{ZmHPs}$ possessed both HPt domains (Figure 1), which illustrates that the HPt is highly conserved in evolution and is vital for histidine phosphotransfer activity.

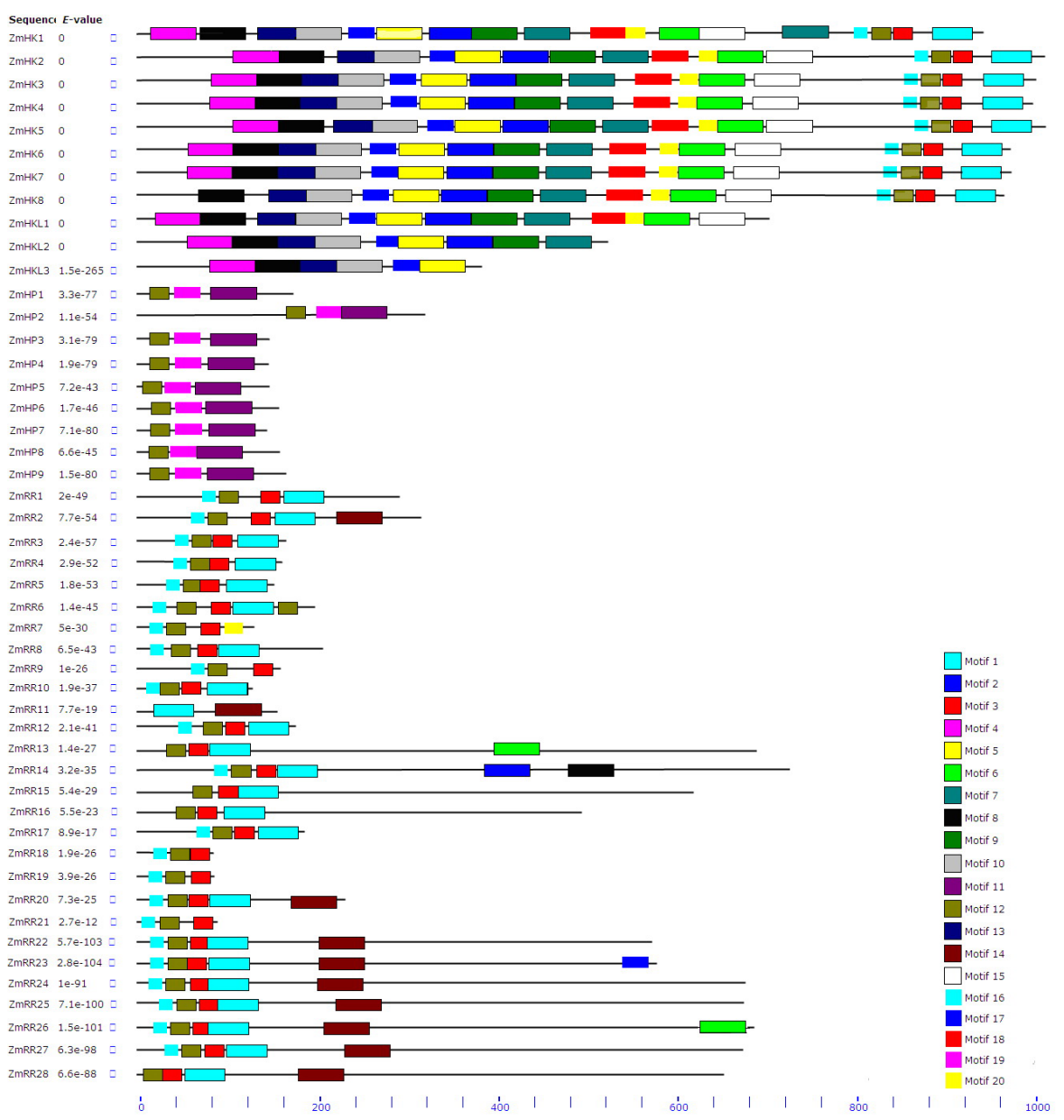

Figure 1. Analysis of primary domain of cytokinin two-component elements in maize. 
The proteins ZmRR1 to ZmRR21 are A-type response regulators while ZmRR22 to ZmRR28 are B-type RRs (Figure 1). While type-A ZmRRs contained a receiver domain with short $\mathrm{N}$ - and C-terminal extensions, type- $\mathrm{Z} Z \mathrm{mRRs}$ contained long $\mathrm{C}$-terminal extensions that mediate sequence-specific DNA binding and transcriptional activation. All the $\mathrm{ZmRRs}$ contained an $\mathrm{RR}$ receiver domain which in orthologues is crucial to accept the phosphoryl group. The type-B RRs could cause induction of ARRs, and the Myb DNA-binding domain in type-B ZmRRs may be useful for this.

\section{Conserved domain analysis in $T C S$ genes}

By using the Pfam database, we deduced the amino acid sequences of conserved domains in ZmHKs, ZmHKLs, ZmHPs, and ZmRRs. We then compared protein sequences by ClustW (Figure 2). All of the ZmHKs and ZmHKLs contained the conserved Thr residue in the CHASE domain (Figure 2A), most likely because the Thr residue is necessary for cytokinin recognition and binding (Mahonen et al., 2000). Thus, all ZmHKs and ZmHKLs recognize and bind cytokinins. The conserved sites in ZmHKs also included a His residue in the HisKA domain. The DDK sequence in the receiver domain was also highly homologous (Figure 2B, C).

The His residue in the HPt domain of ZmHPs, which could be phosphorylated, was conserved except in ZmHP6 and ZmHP8 where it was replaced by Gln (Q) (Figure 2D). The lack of the His residue may confer complete function to ZmHP1-5, ZmHP7, and ZmHP9, while ZmHP6 and ZmHP8 retain only partial function.

The type-A ZmRRs ZmRR1-6 and ZmRR8 had a conserved DDK in the receiver domain which in orthologues are required to accept a phosphoryl group (Schaller et al., 2002), so these two proteins likely function in the phosphorelay. In contrast, ZmRR10, 11, 12, 13, 20, 21, and 17 all lack the first Asp residue (D), ZmRR11, 12, 13, 14, 15, 16, and 20 all lack the second Asp residue (D), while ZmRR7, 9, 18, 19, and 21 all lack the Lys residue (K). Thus, these latter A-type ZmRRs may only retain partial function (Figure 2E). Out of 7 typeB ZmRRs, ZmRR21-27 contained the integral DDK that characterizes the receiver domain, while only ZmRR28 lacked the first Asp residue (D) leading to partial function (Figure 2F). The amino acid sequences of the Myb-like DNA binding motifs of B-ZmRRs were highly conserved (Figure 2G). These results suggest that that the DDK in ZmRR28 may have mutated during evolution. However, no receptor lacked more than one of the three amino acid residues within the DDK motif, suggesting that proteins expressing DD or DK retain partial function.

Phylogenetic analysis of the two-component system genes in maize, Arabidopsis, and rice, $5 \mathrm{AHKs}, 5 \mathrm{OsHKs}$, and $11 \mathrm{ZmHKs}$ ( $8 \mathrm{ZmHKs}$ and $3 \mathrm{ZmHKLs}$ ) were used to construct an unrooted phylogenetic tree of $H K s$ (Figure 3A). The histidine kinase family fell into two groups, I and II. The proteins $Z m H K 1, Z m H K 2, Z m H K 5$, and $Z m H K L 1$ formed clade I, while ZmHK3, ZmHK4, ZmHK6, ZmHK7, ZmHK8, ZmHKL2, and ZmHKL3 formed clade II. In addition, $A H K 2, A H K 3, A H K 4, O s H K 2, O s H K 3$, and $O s H K 5$ were in clade I. In the phylogenetic tree, the $Z m H K s$ and $O s H K s$ were closely related. This may suggest that the common ancestor of the $H K s$ predates the separation of monocots and dicots.

Six $A H P s, 5 O s H P s$, and $9 \mathrm{ZmHPs}$ were selected to construct an unrooted phylogenetic tree of $H P S$ (Figure 3B). The histidine phosphotransfer protein family can be divided into two groups, I and II. All AHPs, along with OsHP3, OsHP4, OsHP5, ZmHP5, ZmHP6, and ZmHP8 formed a clade distinct from the other $O s H P s$ and $Z m H P S$. The results probably indicate that $H P S$ expanded from an ancestral gene that existed prior to the divergence of monocots and dicots. 


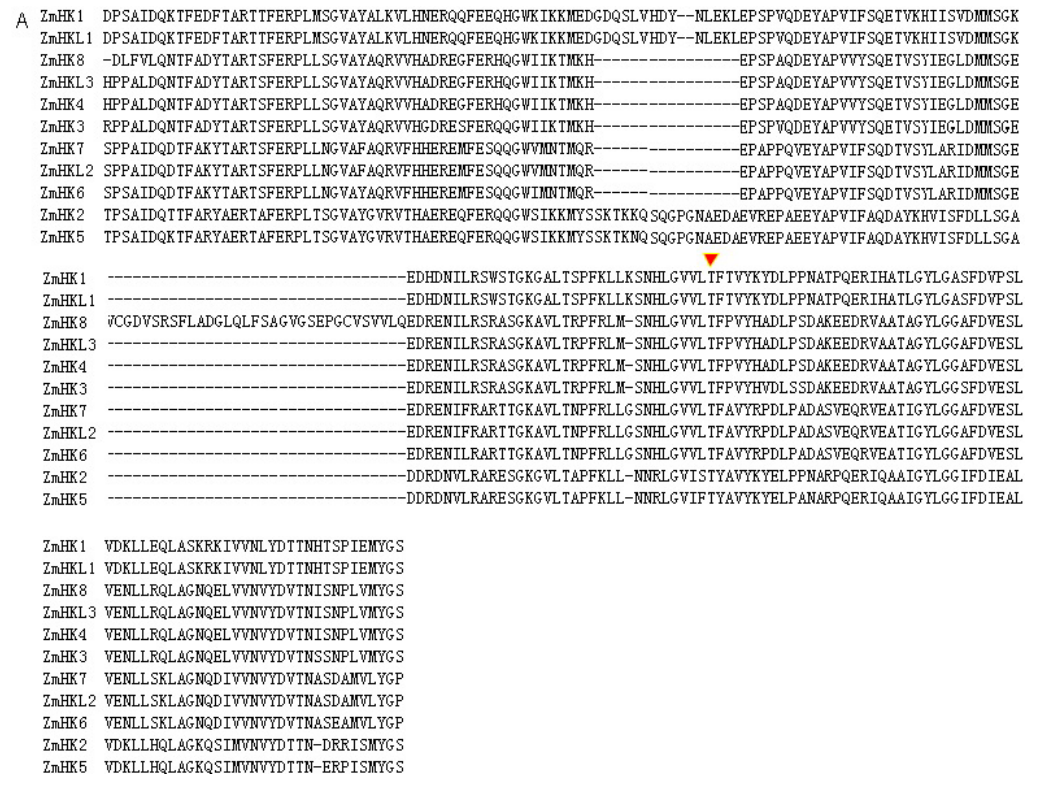

B ZMHK4 AKSQFLATVSHEIRTPMWGVLGMLDMLLDTDLTSTQRDF AQTAQWCGKAL ISL INEVLDRAKIEARKLDLESWPFDLRSILDDVISLFSSKSREKG IELD

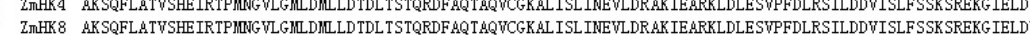

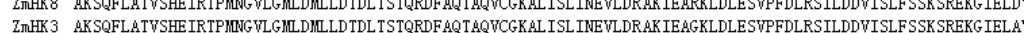

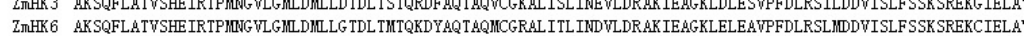

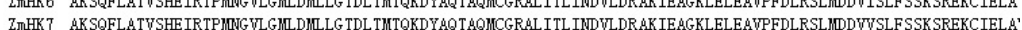

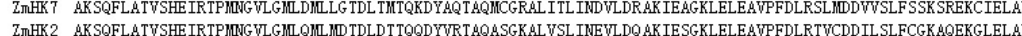

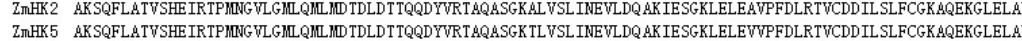
ZMHK 1 AKSQFLATVSHEIRTPMNG VLGILQILID TELDT TQQDFWTAQESGKVL INL INEVLDL AK IESGRIELEAVPFDVRDILDNVISLFYDKSQAKG IELA'

ZMHK4 WVERVPEILLGDPGRFRQIITNL VGNSIKF TERGHIFWQVHLADHSNLATESKVEPVANGMNGHTDEK TAVATSWSLN TLSGFEAGDSRNSWENFKLLI ZMHK8 WSERVPEILLGDPGRFRQIITNL VGNSTKF TERGHIFVQVHLADHSNLATESKVEPVANGIISGHTDEK TAVATSVSLN TLSGFEAADSRNSWENFKLLL ZMHK3 TVSERVPELLLGDPGRFRQIITNL VGNSIKF TERGHIFVQVHLADHSNLATEPKVESVANGMIGGHKDEK TAVATSVSLN TLSGFEAGDSRNSWENFKLLL

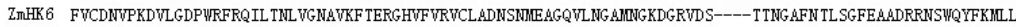
ZMHKT FWCDNWPKWIGDPWRFRQIL TNL WGNAVKF TERGHVFVRWCLAENSNVEANQVLHGMMNGKGGRVES----TTNG FFN TLSGFEAMDRRNSWQYFKLLL ZMHK2 FVSDQVPQQLIGDPGRIRQIITNL VGNSIKF TEKGHIYL TVHWVEIMN----CLEVETG------------TQSANTLSG YPVANRKRSWENFRVFS

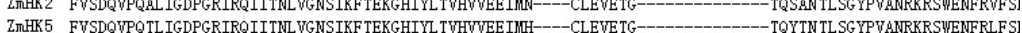

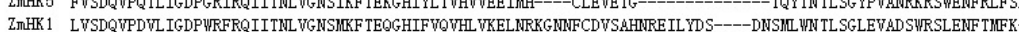

ZMHK4 YEK------NEMPYESWSDKVTLWSVEDTG IGIPLDAQAKVF TPFMQADSSTSRTYGG TG IGLSISKCLVELMGGQINFVSRPHVGSTFTFTAVLK ZMHK8 YEK------NEMPYESVSDKVTLWSVED TG IG IPLDAQAKVF TPFMQADSSTSRTYGG TG IGLSISKCLVELMGGINFVSRPHVGSTF TF TAVL ZMHK 3 YEK------NEMPYESVSDKVTLWSVED TG IG IPLDAQAKVF TPFMQADSSTSRTYGG TG IGLSISKCLVELMGGQINFVSRPHVGSTF TFTAML 7 ZMHKT DKESLLDDLEGENSNQSDSNHVTLAISIEDTGVG IPLQAQDRVF TPFMQADSSTSRNYGG TG IGLSISKCLAELMGGQISF TSHP SVGSTFTFSATLK ZMHK2 ELNSS-----EMPFAPIASDSISLIISVEDTGWG IPFDAQSRVF TPFMQWGPSIARIHGG TG IGLSISKCL WGLMRGEIGFASKPQVGSTF TFTAMU Z ZMHK 1 --NSN----GE-----TDTIRLAVRVED TG IGITKDAQMRIF TPFMQADSSTSRTYGG TG IGLSITKRL VELMGGEIGF TSKSGWGSTFSFTAIFK

Figure 2. Conserved domains within maize TCS genes. A. CHASE domains of ZmHKs and ZmHKLs. The arrow points to the conserved Thr. B. HisKA domains of ZmHKs. The arrow points to the conserved His which could be phosphorylated. C. Receiver domains of ZmHKs. Arrows point to the DDK triplet that is required to accept the phosphoryl group. The third arrow points to the conserved Asp which could be phosphorylated. D. Hpt domains of ZmHPs. The arrow points to the conserved His which could be phosphorylated. E. Receiver domains of type-A ZmRRs. Arrows point to the DDK which is able to accept phosphoryl groups. The third arrow points to the conserved Asp which could be phosphorylated. F. Receiver domains of type-A ZmRRs. Arrows point to the DDK which is required to accept phosphoryl groups. The third arrow points to the conserved Asp which could be phosphorylated. G. Myb-binding domains of type-B ZmRRs.

Continued on next page 
Figure 2. Continued.

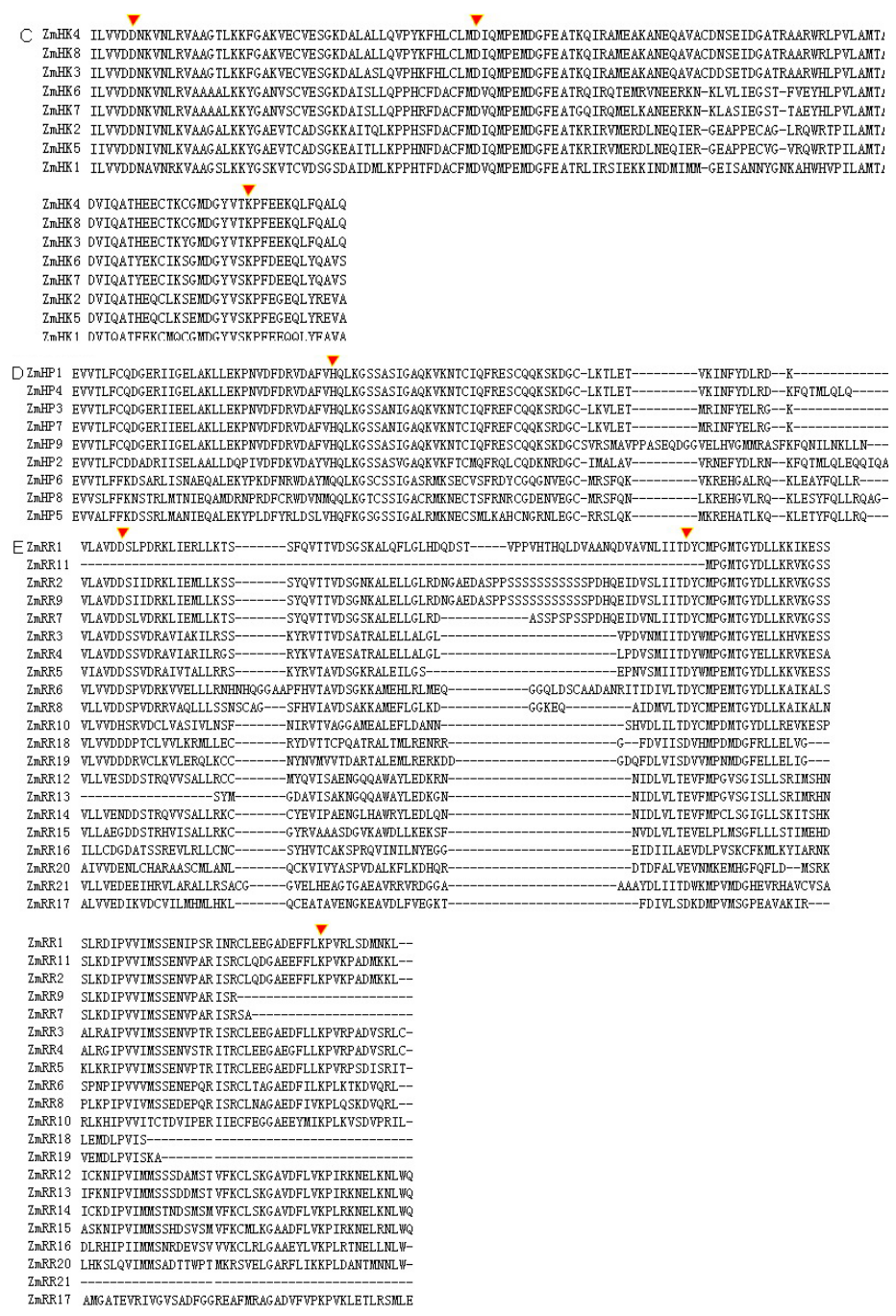

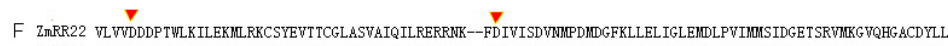

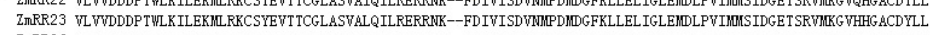
ZMRR26 VL WDDDPTCL WLKRILLECRYDVT TCPQATRAL TILRENRRG--FDVIISDVHIPDNDGFRLLEL VGLERDLPVIMUSSADSRTD IVIKG IKHG ACDYL

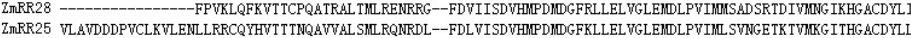

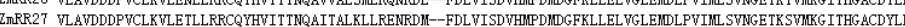
ZMRR24 VLAVDDDRVCLKILERQLKCCNYNT TWTDAQTALDILRERKDGNQFDLVISDWNTKIDGFKLLLLIGLEMDLPVIMLSANSETQTIMKG IKHG ACDYIYY

ZMRR22 KPURIKELRNIWQ

ZMRR23 KPYRMKELRNIWQ

ZMRR26 KPVRMEELKNIWQ

ZMRR28 KPVRIEELKNIWQ

ZMRR25 KPVRLEELRNIWQ

ZMRR27 KPVRIEELRNIWQ

G ZMRR24 RWWYSVELHRKFVAAYWQLG IDKAYPKRILELMNVERL TRENYASHLQKYR ZMRR25 RWWWSVELHRKFVAAWNQLG IDKAVPKRILELMNVERL TRENVASHLQKYR ZMRR27 RWWYSIELHRKFWAAWNQLG IDKAYPKRILELMNVEKL TRENVASHLQKYR ZMRR26 RWWWSVLLQQFWNATNHLG IDKAVPKKILELMNVPGL TRENVASHLQKFR

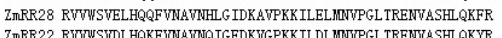

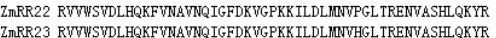


Thirteen A-ARRs, $10 B$-ARRs, 9 A-OsRRs, $21 A$-ZmRRs, and 7 B-ZmRRs were selected to construct an unrooted phylogenetic tree of RRs (Figure 3C). The A-RRs could be divided into three clades while $B$-RRs formed one clade. The type-A ARRs ARR13 and ARR23 are closely related to the type-B $R R s$, and so fall within a clade AII. The rice and maize genes are more closely related to each other than to the RRs from Arabidopsis, indicating that the expansion of $R R$ genes occurred after the divergence of monocot and dicot plants.

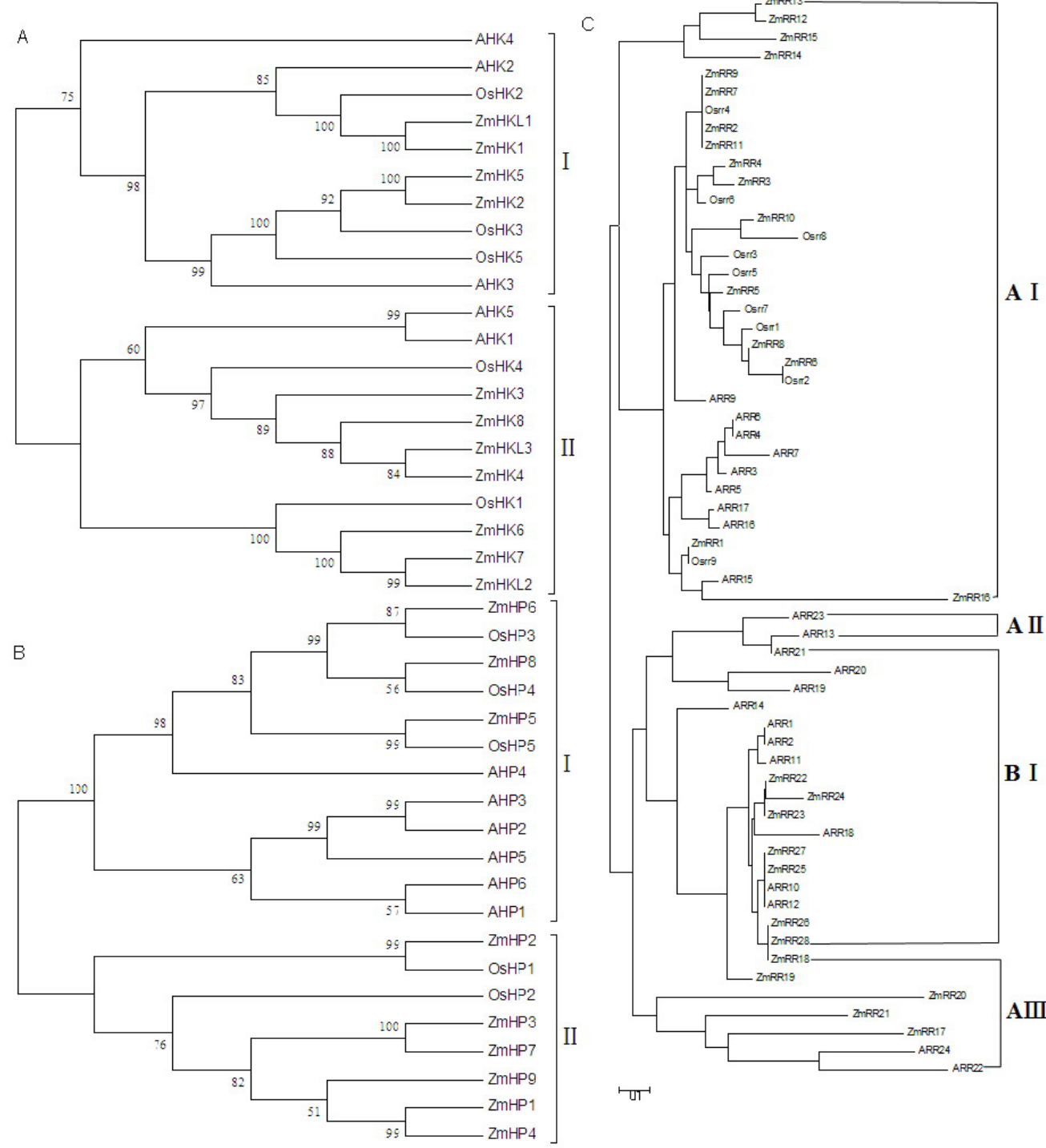

Figure 3. Phylogenetic trees of histidine kinases, histidine phosphotransfer proteins, and response regulators in rice, Arabidopsis, and maize. The phylogenetic trees were constructed using the neighbor-joining method with bootstrap tests by MEGA 4.0. The numbers at the branches are confidence values. A. Histidine kinases. B. Histidine phosphotransfer proteins. C. Response regulators and pseudo-response regulator proteins. $\mathrm{Bar}=0.1$ sequence divergence. 


\section{Distribution and duplication of the two-component genes in the maize genome}

Forty-eight genes that are putative components of the maize cytokinin TCS system were located on the maize chromosomes and their duplication was examined (Figure 4). ZmHK genes were distributed on chromosome 1, 3, 4, 5, and 8. Each chromosome contain only one ZmHK gene, except chromosome 5, which had $7 \mathrm{ZmHK}$ genes. The $9 \mathrm{ZmHP}$ genes were uniformly distributed on chromosome 1, 2, 3, 4, 6, and 8 . The $Z m R R$ genes were concentrated on chromosome 2, which had 5 genes, while chromosomes $1,3,4,5,6,7,9$, and 10 each carried only one. On chromosome 4,5 , and 8 , there were many genes located in close proximity, suggesting that these sites could be hotspots for future research into gene duplication during evolution. We defined a gene duplication according to the following criteria (Gu et al., 2002; Yang et al., 2008): 1) the length of alignable sequence cover $>80 \%$ of the longer gene. 2) the similarity of the aligened regions $>70 \%$. 3) only one event of duplication is counted for tightly linked genes. Each pair of ZmHK1 and ZmHKL1, ZmRR7 and ZmRRL2, ZmHP1 and ZmHP4, and ZmRR2 and ZmRR11 were in tandem repeat. The duplication of these genes reinforces our phylogenetic clustering results. Indeed, we found three groups of genes that shared near total sequence homology within the group (ZmHK4, ZmHK8 and ZmHKL; ZmHP1, 4, and 9; ZmRR2, 9, and 11).

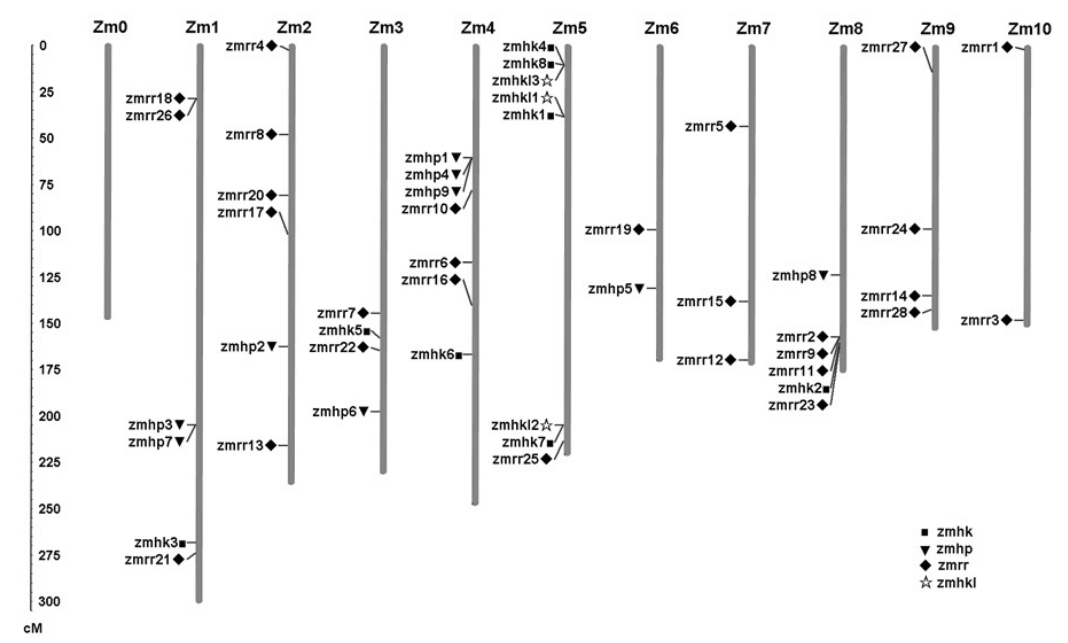

Figure 4. Locations and duplications of putative cytokinin two-component regulators in maize chromosomes.

\section{Expression of $Z m R R$ genes}

The expression pattern of the $Z m R R$ genes ware distributed by RT-PCR. The data from RT-PCR analyses on RNA isolated from leaves and roots of Zea mays B73 seedlings showed that out of the $28 Z m R R$ genes, 27 were expressed in the leaves and 24 in the roots (Figure 5). Most $Z m R R$ genes were expressed both in the leaves and roots except $Z m R R 10, Z m R R 14, Z m R R 16$, $Z m R R 19$, and $Z m R R 23$, which were only expressed in the roots. Conversely, ZmRR10, ZmRR14, $Z m R R 19$, and $Z m R R 23$ could be detected in the leaves but not in the roots. The overall expression of $A-Z m R R s$ was higher than $B-Z m R R s$ both in the leaves and roots, but expression levels were more variable. There was no significant differences in the expression level of individual 
$Z m R R s$ between leaves and roots. These expression data indicate that, although sequence analysis suggested partial function, all 28 genes maybe encode biologically active proteins.

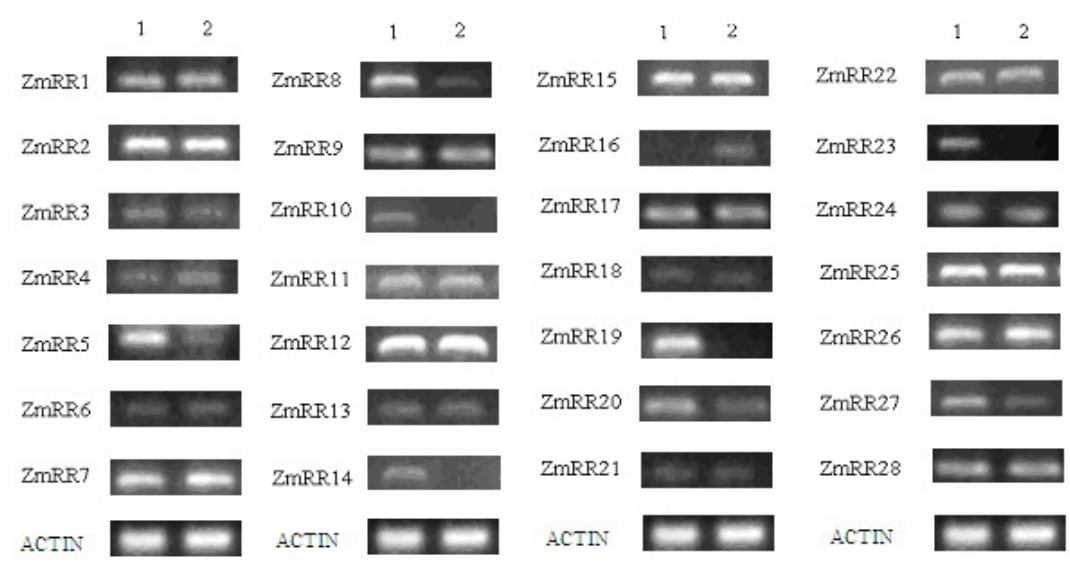

Figure 5. Expression pattern of ZmRR genes. RNAs were isolated from roots and leaves from B73. Lane $1=$ Leaf of B73; lane 2 = Root of B73.

\section{DISCUSSION}

Many of the genes found in the maize two-component signal system have been found in several other species of plants, therefore this pathway may constitute an ubiquitous transduction pathway. We used the sequence data of genes from the TCS of Arabidopsis and rice as queries to find the maximum number of putative TCS genes in maize.

The sequences of the TCS genes from Arabidopsis and rice were downloaded from the NCBI database and used as queries for the maize genomic work. The BLAST search yielded 48 maize TCS genes, including 11 ZmHKs (ZmHK1-8, ZmHKL1-3), nine ZmHPs, 21 type-A ZmRRs, and seven type-B ZmRRs. The 11 ZmHKs all contained a conserved CHASE domain at the N-terminus which was followed by a transmitter and a receiver domain in all except ZmHKL1-3 (which only containing the CHASE domain). These domain sequences suggest that ZmHK1-8 act as ligand-activated histidine kinases while ZmHKL1-3 may have lost the cytokinin receptor function. Obviously, the CHASE domain is functionally irreplaceable. Conserved domains within the different ZmHPs and ZmRRs were highly homologous, as where domains within OsHPs, OsRRs, AHPs, and ARRs, illustrating that the downstream signaling elements of the two-component system were also highly conserved during evolution.

ZmHK1-8 contained a conserved Thr residue in the CHASE domain, a conserved His residue in the activity domain, and a DDK triplet in the receiver domain. Such high conservation was not found in the ZmHPs and ZmRRs, which suggesting that the ZmHPs and ZmRRs may have function redundancy, while every $\mathrm{ZmHK}$ is crucial for cytokinin signaling.

The homologous gene clusters illustrated in the phylogenetic trees of the maize TCS genes indicate an extensive number of gene duplications throughout evolution. Three gene pairs were found as tandem repeats (ZmHK1 and ZmHKL1, ZmRR7 and ZmRRL2, ZmHP1 and ZmHP4, and $Z m R R 2$ and $Z m R R 11)$, while in general, ZmHK, ZmHP, and ZmRR genes had high densities on spe- 
cific chromosomes $\left(5,4\right.$, and 2 respectively). In contrast, $O_{s} H K$ and $O s H P$ genes were distributed uniformly (Du et al., 2007; Ildoo et al., 2002). Analysis of the duplicate genes sequences suggested that there was gene duplication and a certain extent of variation in the evolutionary process.

The expression of the $Z m R R$ genes was detected by RT-PCR. The results showed some tissue-specific expression; for example $Z m R R 16$ could be detected in the roots but not in the leaves, while $Z m R R 10, Z m R R 14, Z m R R 19$, and $Z m R R 23$ were detected in the leaves but not in the roots. Most other $Z m R R$ genes were expressed in both leaves and roots. As another species of dicotyledon, the expression of the OsRR genes in rice was also examined by RT-PCR. The expression of most type-A and type-B OsRR genes was detected in leaves and roots, except for OsRR11, which was expressed only in roots. However, three type-A OsRR genes, OsRR8, OsRR12, and OsRR 13, and one type-B OsRR gene, OsRR19, were not detected in leaves and roots. The expression of type-B OsRR genes was higher in both leaves and roots compared with type-A OsRR genes. Thus, region-specific expression was apparent in both species of dicotyledon.

We examined maize TCS genes by bioinformatics, a task greatly facilitated by the recent publication of the B73 maize genome (Schnable et al., 2009). Though there are many of studies of TCS genes in both Arabidopsis and rice, this is the first comprehensive analysis of maize and may guide future research in this species. Finally, although the two-component signaling system is very important in regulating responses to cytokinin in different tissues and at different stages of plant development, alternative pathways (Romanov et al., 2002) may also contribute to growth and development.

\section{ACKNOWLEDGMENTS}

Research supported by the National Key Project of Transgenic Crops of China (\#2008ZX003-002 and \#2008ZX08010-001) and the National Technology Support Project (\#2009BADA6B00).

\section{REFERENCES}

Aoyama T and Oka A (2003). Cytokinin signal transduction in plant cells. J. Plant Res. 116: 221-231.

Asakura Y, Hagino T, Ohta Y, Aoki K, et al. (2003). Molecular characterization of His-Asp phosphorelay signaling factors in maize leaves: implications of the signal divergence by cytokinin-inducible response regulators in the cytosol and the nuclei. Plant Mol. Biol. 52: 331-341.

Bailey TL, Williams N, Misleh C and Li WW (2006). MEME: discovering and analyzing DNA and protein sequence motifs. Nucleic Acids Res. 34: W369-W373.

Brandstatter I and Kieber JJ (1998). Two genes with similarity to bacterial response regulators are rapidly and specifically induced by cytokinin in Arabidopsis. Plant Cell 10: 1009-1019.

D’Agostino IB and Kieber JJ (1999). Phosphorelay signal transduction: the emerging family of plant response regulators. Trends Biochem. Sci. 24: 452-456.

D'Agostino IB, Deruere J and Kieber JJ (2000). Characterization of the response of the Arabidopsis response regulator gene family to cytokinin. Plant Physiol. 124: 1706-1717.

Du L, Jiao F, Chu J, Jin G, et al. (2007). The two-component signal system in rice (Oryza sativa L.): a genome-wide study of cytokinin signal perception and transduction. Genomics 89: 697-707.

Forde BG (2002). Local and long-range signaling pathways regulating plant responses to nitrate. Annu. Rev. Plant Biol. 53: 203-224.

Grefen C and Harter K (2004). Plant two-component systems: principles, functions, complexity and cross talk. Planta 219: 733-742.

Gu Z, Cavalcanti A, Chen FC, Bouman P, et al. (2002). Extent of gene duplication in the genomes of Drosophila, nematode, and yeast. Mol. Biol. Evol. 19: 256-262.

Hass C, Lohrmann J, Albrecht V, Sweere U, et al. (2004). The response regulator 2 mediates ethylene signalling and 
hormone signal integration in Arabidopsis. EMBO J. 23: 3290-3302.

Hutchison CE and Kieber JJ (2002). Cytokinin signaling in Arabidopsis. Plant Cell 14: S47-S59.

Hwang I and Sheen J (2001). Two-component circuitry in Arabidopsis cytokinin signal transduction. Nature 413: 383-389. Hwang I, Chen HC and Sheen J (2002). Two-component signal transduction pathways in Arabidopsis. Plant Physiol. 129: 500-515. Ildoo H, Huei-Chi C and Jen S (2002). Two-component signal transduction pathways in Arabidopsis. Plant Physiol. 129: 500-515. Inoue T, Higuchi M, Hashimoto Y, Seki M, et al. (2001). Identification of CRE1 as a cytokinin receptor from Arabidopsis. Nature 409: 1060-1063.

Lohrmann J, Buchholz G, Keitel C, Sweere U, et al. (1999). Differential expression and nuclear localization of response regulator-like proteins from Arabidopsis thaliana. Plant Biol. 1: 495-505.

Lohrmann J, Sweere U, Zabaleta E, Baurle I, et al. (2001). The response regulator ARR2: a pollen-specific transcription factor involved in the expression of nuclear genes for components of mitochondrial complex I in Arabidopsis. Mol. Genet. Genomics 265: 2-13.

Mahonen AP, Bonke M, Kauppinen L, Riikonen M, et al. (2000). A novel two-component hybrid molecule regulates vascular morphogenesis of the Arabidopsis root. Genes Dev. 14: 2938-2943.

Martín AC, del Pozo JC, Iglesias J, Rubio V, et al. (2000). Influence of cytokinins on the expression of phosphate starvation responsive genes in Arabidopsis. Plant J. 24: 559-567.

Mason MG, Mathews DE, Argyros DA, Maxwell BB, et al. (2005). Multiple type-B response regulators mediate cytokinin signal transduction in Arabidopsis. Plant Cell 17: 3007-3018.

Mok DW and Mok MC (2001). Cytokinin metabolism and action. Annu. Rev. Plant Physiol. Plant Mol. Biol. 52: 89-118.

Pischke MS, Jones LG, Otsuga D, Fernandez DE, et al. (2002). An Arabidopsis histidine kinase is essential for megagametogenesis. Proc. Natl. Acad. Sci. U. S. A. 99: 15800-15805.

Riechmann JL, Heard J, Martin G, Reuber L, et al. (2000). Arabidopsis transcription factors: genome-wide comparative analysis among eukaryotes. Science 290: 2105-2110.

Riefler M, Novak O, Strnad M and Schmulling T (2006). Arabidopsis cytokinin receptor mutants reveal functions in shoot growth, leaf senescence, seed size, germination, root development, and cytokinin metabolism. Plant Cell 18: 40-54.

Romanov GA, Kieber JJ and Schmulling T (2002). A rapid cytokinin response assay in Arabidopsis indicates a role for phospholipase D in cytokinin signalling. FEBS Lett. 515: 39-43.

Sakai H, Aoyama T and Oka A (2000). Arabidopsis ARR1 and ARR2 response regulators operate as transcriptional activators. Plant J. 24: 703-711.

Schaller GE, Mathews DE, Gribskov M and Walker JC (2002). Two-Component Signalling Elements and Histidyl-Aspartyl Phosphorelays. In: The Arabidopsis book American Society of Plant Biologists (Somerville C and Meyerowitz E, eds.). DOI/10.1199/tab.0086, Available at [http:/www.aspb.org/publications/Arabidopsis]. Accessed......

Schnable PS, Ware D, Fulton RS, Stein JC, et al. (2009). The B73 maize genome: complexity, diversity, and dynamics. Science 326: 1112-1115.

Stock AM, Robinson VL and Goudreau PN (2000). Two-component signal transduction. Annu. Rev. Biochem. 69: 183-215.

Suzuki T, Miwa K, Ishikawa K, Yamada H, et al. (2001). The Arabidopsis sensor His-kinase, AHk4, can respond to cytokinins. Plant Cell Physiol. 42: 107-113.

Thomason P and Kay R (2000). Eukaryotic signal transduction via histidine-aspartate phosphorelay. J. Cell Sci. 113: 3141-3150.

Thompson JD, Higgins DG and Gibson TJ (1994). CLUSTAL W: improving the sensitivity of progressive multiple sequence alignment through sequence weighting, position-specific gap penalties and weight matrix choice. Nucleic Acids Res. 22: 4673-4680.

To JP, Haberer G, Ferreira FJ, Deruere J, et al. (2004). Type-A Arabidopsis response regulators are partially redundant negative regulators of cytokinin signaling. Plant Cell 16: 658-671.

Ueguchi C, Koizumi H, Suzuki T and Mizuno T (2001). Novel family of sensor histidine kinase genes in Arabidopsis thaliana. Plant Cell Physiol. 42: 231-235.

Urao T, Yakubov B, Yamaguchi-Shinozaki K and Shinozaki K (1998). Stress-responsive expression of genes for twocomponent response regulator-like proteins in Arabidopsis thaliana. FEBS Lett. 427: 175-178.

West AH and Stock AM (2001). Histidine kinases and response regulator proteins in two-component signaling systems. Trends Biochem. Sci. 26: 369-376.

Yamada S and Shiro Y (2008). Structural basis of the signal transduction in the two-component system. Adv. Exp. Med. Biol. 631: 22-39.

Yang S, Zhang X, Yue JX, Tian D, et al. (2008). Recent duplications dominate NBS-encoding gene expansion in two woody species. Mol. Genet. Genomics 280: 187-198.

Yonekura-Sakakibara K, Kojima M, Yamaya T and Sakakibara H (2004). Molecular characterization of cytokinin-responsive histidine kinases in maize. Differential ligand preferences and response to cis-zeatin. Plant Physiol. 134: 1654-1661. 


\section{SUPPLEMENTARY MATERIAL}

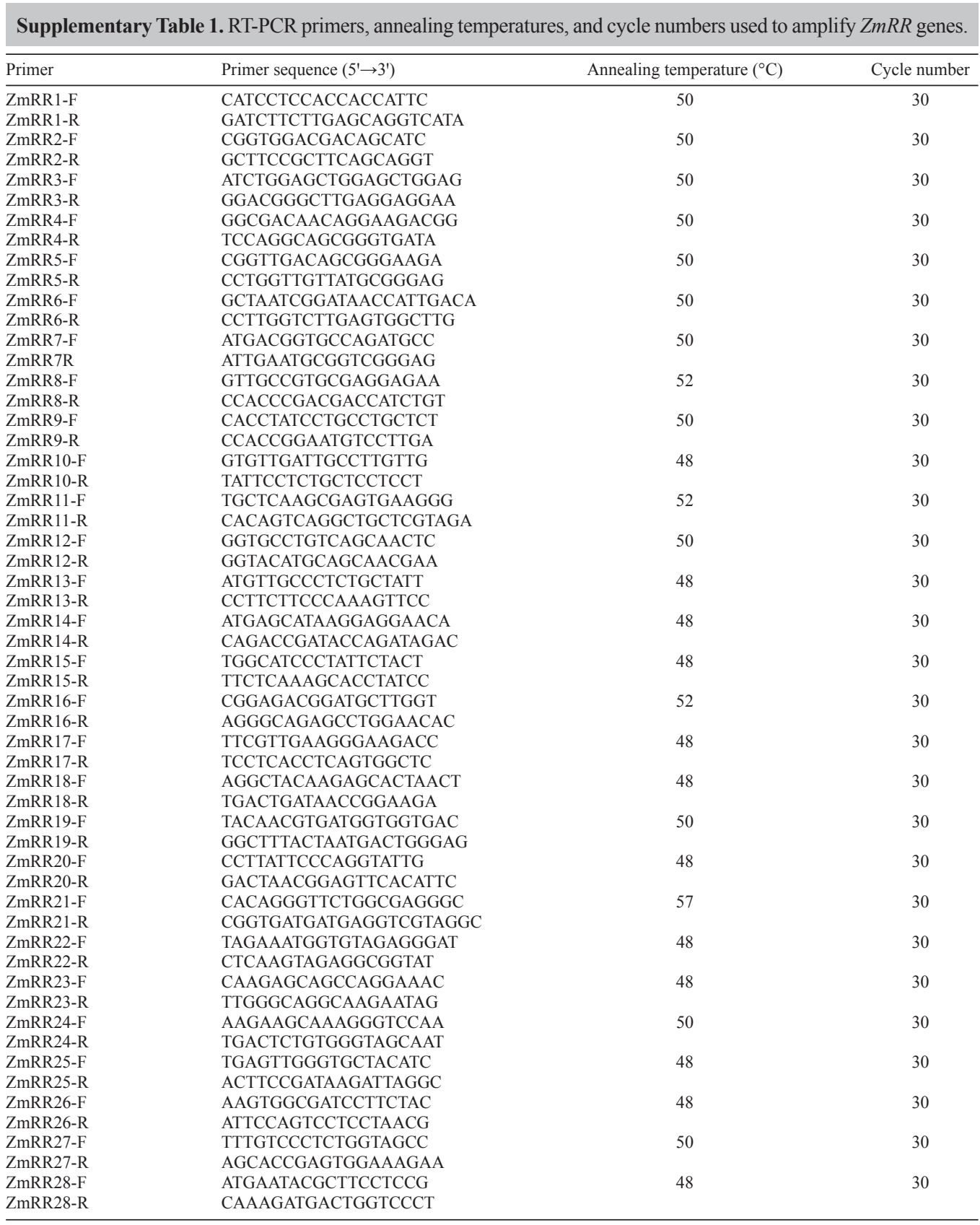

